\title{
Disfagia orofaríngea no idoso
}

Luiz João Abrahão Júnior

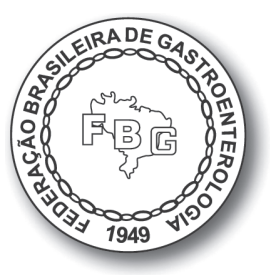

\section{INTRODUÇÃO}

Disfagia orofaríngea (DOF) é uma condição caracterizada por dificuldade real ou percebida em formar ou mover o bolo alimentar de maneira segura da cavidade oral para o esôfago. A DOF deve ser diferenciada de distúrbios alimentares associados com alterações dos hábitos normais de alimentação e do globo faríngeo, que é sensação de bolo na garganta, sem dificuldade de deglutição.

\section{EPIDEMIOLOGIA}

Estima-se que, na população geral, a prevalência da DOF varie entre 2,3 e $16 \%$, dados estes obtidos em consultas telefônicas. Esta prevalência aumenta com a idade, atingindo $26,7 \%$ em indivíduos acima dos 76 anos. Tem sido estimado que ocorra em 30 a $40 \%$ de pessoas idosas independentes, em $44 \%$ daqueles necessitados de cuidados intensivos e em $60 \%$ de idosos institucionalizados.

As consequências potenciais da DOF em idosos são desidratação/desnutrição, pneumonias por aspiração, perda do prazer com a alimentação e isolamento familiar e social.

\section{ETIOLOGIA}

Na Tabela 1, estão as principais causas de DOF. É mais comum em idosos, sendo causas frequentes a DOF pós-acidente vascular cerebral (AVC) e a associada à doença de Parkinson (PK). Alterações anatômicas, fisiológicas, psicológicas e funcionais que contribuem para anormalidades na deglutição 
como parte do envelhecimento são intituladas de "presbifagia" e promovem redução natural da reserva funcional. Embora estas alterações sejam progressivas e coloquem idosos em risco de DOF, a deglutição em idosos saudáveis não é necessariamente comprometida.

A DOF em idosos com demência e/ou doença de Alzheimer pode atingir $93 \%$, com até $28 \%$ apresentando aspiração quando investigados. Em pessoas com menos de 60 anos, a DOF é mais frequentemente associada a doenças oncológicas com ou sem radioterapia e a doenças neurológicas degenerativas.

\begin{tabular}{|c|c|}
\hline \multirow{6}{*}{$\begin{array}{l}\text { Sistema nervoso } \\
\text { central }\end{array}$} & Acidente vascular cerebral \\
\hline & $\begin{array}{l}\text { Sindrome extrapiramidal (Parkinson, coreia de Huntington, doença de } \\
\text { Wilson) }\end{array}$ \\
\hline & Tumores do tronco cerebral \\
\hline & Doença de Alzheimer \\
\hline & Esclerose lateral amiotrófica \\
\hline & Drogas \\
\hline \multirow{4}{*}{$\begin{array}{l}\text { Sistema nervoso } \\
\text { periférico }\end{array}$} & Atrofia muscular espinal \\
\hline & Síndrome de Guillain-Barré \\
\hline & Síndrome pós-poliomielite \\
\hline & Drogas (procainamida, citotóxicos) \\
\hline \multirow[t]{5}{*}{ Miogênica } & Miastenia grave \\
\hline & Dermatomiosite, polimiosite \\
\hline & Miopatia tireotóxica \\
\hline & Síndrome paraneoplásica \\
\hline & Drogas (amiodarona, álcool, drogas redutoras de colesterol) \\
\hline \multirow{6}{*}{$\begin{array}{l}\text { Alterações } \\
\text { estruturais }\end{array}$} & Divertículo de Zenker \\
\hline & Barra ou estenose do cricofaríngeo \\
\hline & Anel cervical \\
\hline & Tumores de orofaringe \\
\hline & Cirurgia de cabeça e pescoço \\
\hline & Radioterapia \\
\hline
\end{tabular}

\section{FISIOPATOLOGIA}

$\mathrm{O}$ trato digestivo superior realiza duas funções na mesma via anatômica: a respiração e a deglutição. A deglutição orofaríngea depende da configuração das vias digestiva e respiratória na passagem do alimento da cavidade oral para 
o esôfago. Este processo complexo, que envolve componentes sensitivos, motores e psicológicos, inclui mais de 40 músculos e 6 pares cranianos (V, VII, IX, $\mathrm{X}, \mathrm{XI}$ e XII), pode ser descrito em três fases sequenciais:

- Fase oral preparatória, que dispõe de ações voluntárias e reflexas. A mastigação torna o alimento homogêneo, o qual se dirige para a parte posterior da boca, impulsionado pelos movimentos superior e posterior da língua.

- Fase faríngea, involuntária, em que o palato mole se eleva para fechar a nasofaringe e prevenir a regurgitação nasal. $\mathrm{O}$ osso hioide ascende, elevando a laringe, enquanto a epiglote fecha sua entrada, o que coincide com o relaxamento do músculo cricofaríngeo e abertura do esfíncter esofágico superior (EES).

- Fase esofágica, também involuntária, em que o alimento, uma vez ultrapassado o EES, segue para o estômago, impulsionado pela peristalse esofágica primária. A duração do ato da deglutição em um indivíduo saudável é de 0,6 a 1 segundo.

\section{QUADRO CLÍNICO}

A faixa etária em que a DOF se apresenta varia com a etiologia, porém, como as doenças neurológicas são a causa mais frequente, costuma surgir após os 60 anos.

Os sintomas relacionam-se com o ato de deglutir, e a anamnese pode sugerir o diagnóstico. Os pacientes referem dificuldade de deglutição apontando a região cervical (disfagia alta). A dificuldade, com frequência, é acompanhada de engasgos, tosse e, algumas vezes, de regurgitação de líquidos pelas fossas nasais. Por essa razão, as refeições são longas, o que não raramente afasta os pacientes do convívio familiar. Com o prolongamento do quadro, podem ocorrer perda de peso e desnutrição, o que piora o prognóstico destes pacientes, frequentemente portadores de comorbidades. A possibilidade de aspiração pode levar ao desenvolvimento de pneumonias. Sabe-se que metade dos portadores de DOF com aspiração vai a óbito em torno de 1 ano. É frequente o paciente "alimentar-se tossindo", sugerindo, pelo menos, tentativa de proteção das vias aéreas. A retenção de saliva ou resíduos na faringe gera a alteração vocal conhecida como "voz úmida". Fala anasalada com sucessivas tentativas de melhorá-la são comuns. Sintomas e sinais neurológicos da doença de base acompanham o quadro, como os da doença de Parkinson ou sequelas de AVC, além de alterações mentais mais ou menos acentuadas nos casos de demência e/ou doença de Alzheimer. 
Aspectos emocionais e psicológicos devem ser lembrados. Sensação de pânico ao se alimentar é observada em $40 \%$ dos pacientes, enquanto $36 \%$ evitam se alimentar em companhia de outras pessoas. Ansiedade e depressão podem ser encontradas em $37 \%$ e $32 \%$ dos pacientes, respectivamente. Muitos fazem uso de medicações que podem contribuir para ou até mesmo ser a causa da disfagia, como anticolinérgicos, benzodiazepínicos, haloperidol e inibidores da recaptação de serotonina.

As consequências da DOF, como desidratação, emagrecimento, má nutrição e infecções, principalmente respiratórias, conduzem a internações, readmissões e finalmente a institucionalizações. O exame físico mostra sinais de perda de peso, desidratação, má nutrição e achados da doença neurológica de base, caso seja esta a causa da DOF. Na Tabela 2, estão as manifestações clínicas da DOF.

Tabela 2 Quadro clínico da disfagia orofaríngea

\begin{tabular}{ll} 
Apresentação típica & Apresentação atípica \\
Disfagia alta de transferência & Fala anasalada ou molhada \\
\hline Tosse/engasgos & Mudança vocal \\
\hline Refeições longas & Tosse/pigarro ao se alimentar \\
\hline Eliminação de saliva & Dispneia \\
\hline Desidratação & Pneumonia de aspiração \\
\hline Perda de peso & \\
\hline Desnutrição & \\
\hline
\end{tabular}

\section{DIAGNÓSTICO}

A DOF é uma condição de abordagem multidisciplinar envolvendo avaliações clínica ou geriátrica, neurológica, nutricional, otorrinolaringológica, fonoaudilógica, fisioterápica, psicológica e de habilidades funcionais. A atuação destes profissionais deve ocorrer em conjunto para que se obtenham resultados satisfatórios. O diagnóstico clínico pode ser feito pela tomada cuidadosa da história, inclusive com auxílio dos familiares do paciente. O exame físico da cavidade oral, observando-se elevação do palato e movimentos da língua, além da adequada elevação e anteriorização do osso hioide, fornece informações importantes. Devem ser avaliadas a eficiência da deglutição, ou seja, a capacidade de consumir as calorias adequadas para sua nutrição e hidratação e a segurança da deglutição, sem que complicações respiratórias ocorram. Há testes de avaliação da eficácia e segurança da deglutição, como deglutição de água em diferentes volumes, observando-se surgimento de tosse, voz "úmida" ou rouca 
e administração sequencial de três viscosidades diferentes (néctar, líquido e pudim), explorando os mesmos quesitos. Importante a avaliação do sensório antes da realização destes testes, devendo o paciente estar consciente e alerta.

O diagnóstico diferencial deve ser feito com a disfagia esofágica, em que o paciente pode apontar a fúrcula esternal como o local da sensação de parada do alimento, mas não apresenta engasgos ou tosse, e a dificuldade é no transporte do alimento, e não sua transferência.

\section{MÉTODOS COMPLEMENTARES}

$\mathrm{Na}$ avaliação instrumental, deve-se levar em consideração a possibilidade de utilizar seu resultado no planejamento da reabilitação do paciente. Um exame instrumental pode ser indicado para confirmar o diagnóstico, avaliar a segurança da dieta via oral e planejar o tratamento em pacientes com DOF. Os achados são úteis para a compreensão da fisiopatologia das anormalidades, para avaliar a progressão natural da doença e a resposta ao tratamento. As indicações e contraindicações devem levar em consideração o status cognitivo-linguístico e o estado geral e nutricional do paciente.

Os principais métodos de avaliação instrumental são a esofagografia, a videofluoroscopia da deglutição (VFD), a endoscopia digestiva alta, a avaliação endoscópica funcional da deglutição, conhecida pela sigla em inglês FEES (functional efficacy evaluation of swallow) e a esofagomanometria (EMN), com seu sucedâneo, a videomanometria (Tabela 3). A Sociedade Europeia para Distúrbios da Deglutição (ESSD) sugere que pacientes com DOF ou anormalidade nos mecanismos de proteção de vias aéreas devam ter avaliação instrumental com VFD ou FEES, realizadas de maneira padronizada por profissional experiente, porém existem vários protocolos e não há consenso a respeito do número de reavaliações, volume e consistência dos bolus.

\section{Tabela 3 Métodos complementares}

Esofagografia/videofluoroscopia da deglutição

Endoscopia digestiva alta/avaliação endoscópica funcional da deglutição (FEES)

Esofagomanometria ou manometria de alta resolução

Videomanometria

\section{Esofagografia/videofluoroscopia da deglutição (VFD)}

A esofagografia tem limitações na fase inicial da deglutição, em virtude da rapidez dos fenômenos nesta área, porém, por sua maior disponibilidade, pode 
iniciar a investigação, sendo útil no diagnóstico de divertículo de Zencker, barra faríngea e lesões orgânicas, como estenoses e tumores.

A videofluoroscopia (VFD) é um método radiológico não invasivo, que registra em tempo real a dinâmica das fases da deglutição, sendo considerado o método que mais subsídios oferece ao estudo, em especial, das fases oral e faríngea da deglutição. As doses de radiação necessárias ao estudo da deglutição (produto dose-área - DAP) são significativamente baixas na relação custo-benefício e, além da possibilidade de análise qualitativa dos fenômenos registrados, permite a quantificação em dimensão e tempo das estruturas e eventos. Os vídeos da VFD devem ser examinados em ambiente escuro, para reduzir erros de interpretação, e devem mostrar lábios, boca, faringe, coluna cervical e esôfago. As imagens permitem que seja avaliada a eficiência do preparo, da organização e da ejeção do bolo, assim como, na faringe, a presença de escapes do conteúdo da oro para a rinofaringe. $\mathrm{O}$ trânsito faríngeo, a efetividade da abertura da transição faringoesofágica e a eficiência dos mecanismos de proteção das vias aéreas são bem observados e, quando comprometidos, caracterizam-se por penetração e/ou aspiração. A VFD pode ser bastante útil na observação da deglutição, inclusive com execução das manobras facilitadoras. Experiência preliminar com o método avaliou 26 pacientes com DOF no HUCFF-UFRJ ${ }^{1}$. O exame foi anormal em 92\% dos pacientes, chamando atenção a multiplicidade das alterações, como perda de dentes, escape intraoral, resíduos pós-deglutição e anteriorização ruim do hioide.

\section{Endoscopia digestiva alta/avaliação endoscópica funcional da deglutição (FEES)}

A endoscopia digestiva alta (EDA), por sua disponibilidade, frequentemente inicia a investigação de uma DOF, sendo de bastante utilidade na exclusão de alterações orgânicas ou o seu diagnóstico, quando permite observar a lesão e biopsiá-la para diagnóstico definitivo. A EDA também tem grande importância na terapêutica em alguns casos (vide adiante).

A avaliação endoscópica funcional da deglutição (fiberoptic endoscopic evaluation of swallow - FEES) permite a visão direta das estruturas e da dinâmica faríngea com o emprego do videofibroscópio de fibra óptica e anestesia local. De introdução nasal, sua pequena espessura não interfere significativamente na dinâmica palatal. A estrutura faríngea, a morfologia do ádito laríngeo, a estrutura e a dinâmica das pregas vocais podem ser diretamente observadas durante e depois da deglutição. A dinâmica de abertura e fechamento da transição faringoesofágica também pode ser estimada. É possível visualizar 
resíduos nos seios piriformes e nas valéculas, perda prematura ou não controlada de líquidos, penetração, aspiração e deglutições em porções. Um de seus maiores ganhos é a possibilidade de definir a capacidade reflexa de proteção das vias aéreas, com o emprego de pulso de ar ou leve toque nas paredes que delimitam o espaço interaritenoide. Com o uso de corantes, pode-se analisar a eficácia da proteção das vias aéreas. O exame é bem tolerado, fácil de realizar, passível de repetição, além de ser realizável à beira de leito. Sua maior inconveniência é a limitação de sua observação à fase faríngea.

\section{Esofagomanometria}

A esofagomanometria (EMN) pode quantificar a contração da faringe, detectar falha no relaxamento do EES e a coordenação entre a contração faríngea e a abertura do EES. É importante ter parâmetros relacionados à idade ao considerar as alterações. Ao analisar o corpo esofágico e o esfíncter inferior, a EMN pode mostrar anormalidades associadas ou até mudar o diagnóstico. Cerca de $30 \%$ dos pacientes com disfagia esofágica apontam seu desconforto na região cervical, confundindo o diagnóstico.

\section{Videomanometria ou manofluorografia}

A esofagomanometria pode ser combinada à fluoroscopia (videomanometria ou manofluorografia), técnica que permite a correlação entre a movimentação das estruturas anatômicas com a pressão intraluminar e a identificação da pressão intrabolus, que é medida indireta da complacência do EES. O encontro de falha no relaxamento e/ou a elevada pressão intrabolus podem ajudar no diagnóstico e na conduta, particularmente na indicação de miotomia do cricofaríngeo. Os trabalhos na literatura são escassos e são focados no potencial da técnica, ainda restrita a centros de pesquisa.

\section{TRATAMENTO}

Ver Tabela 4.

\section{Medidas gerais}

A estratégia empregada depende da gravidade do caso, se a DOF é aguda ou crônica, de sua intensidade e do diagnóstico. Causas passíveis de tratamento clínico devem receber terapêutica apropriada, como doenças do colágeno, tireoidopatias e algumas doenças neurológicas (Parkinson, miastenia grave). $\mathrm{O}$ 
tratamento leva em consideração o estado geral do paciente, se será ambulatorial ou com paciente internado ou institucionalizado.

Em linhas gerais, as seguintes estratégias podem ser adotadas: comer lentamente e em volumes pequenos (usar colher); concentrar-se somente na deglutição, eliminando distrações; evitar misturar líquidos e sólidos na mesma ingestão; colocar o alimento no lado mais "forte" da boca, se houver fraqueza unilateral; alternar líquidos e sólidos para permitir o escoamento dos resíduos; e contar com auxílio de cuidadores se houver limitações físicas e/ou cognitivas.

A higiene oral deve ser avaliada, pois sua deficiência é fator de risco para pneumonias em idosos com DOF, em virtude da alta prevalência de patógenos na boca. É necessária a verificação da quantidade e da qualidade dos dentes, além de eficácia e ajustamento das próteses dentárias para que a mastigação possa ser satisfatória. É fundamental recomendar a escovação dentária e a limpeza das próteses após cada refeição e um profissional de saúde bucal deve ser consultado periodicamente. Mínima higiene oral deve ser realizada a cada 12 horas, além de bochechos com soluções antissépticas adequadas.

\section{Medidas compensatórias}

Objetivam evitar ou reduzir os efeitos da DOF. A modificação da consistência de sólidos e/ou líquidos é a principal medida compensatória empregada para portadores de DOF e o efeito terapêutico desta estratégia é elevado. O emprego de agentes espessantes é frequente e seu efeito é viscosidade dependente. Os espessantes reduzem a penetração no vestíbulo laríngeo e a aspiração traqueobrônquica, porém a taxa de adesão ao seu uso é baixa (48 a 56\%), por causa de sua textura e seu gosto, do grande esforço na deglutição e da dificuldade de preparar a refeição.

Posturas e manobras durante a deglutição são fáceis de aprender, não requerem grande esforço e podem direcionar o bolus por meio de ajustes biomecânicos. Uma das técnicas é deglutir em posição sentada, com a cabeça fletida e/ou girada para o lado parético, se for o caso. Esta postura "chin down" é fácil de realizar e ajuda a fechar a via respiratória. A manobra de Mendelsohn consiste em elevação manual da laringe para a mais alta posição possível por poucos segundos durante a deglutição, contraindo voluntariamente a musculatura. Deglutição com esforço aumenta o movimento da base da língua, fortalecendo a propulsão do bolus. As deglutições supraglótica e super-supraglótica consistem em respirar profundamente, prender a respiração durante a deglutição e tossir logo após para eliminar resíduos. Estas manobras protegem as vias aéreas por fechamento das pregas vocais antes e durante a deglutição. A diferença entre elas está no nível de esforço em prender a respiração antes da deglutição. 
Tabela 4 Tratamento da disfagia orofaríngea

\begin{tabular}{l} 
1. Causas de tratamento clínico \\
\hline 2. Causas de tratamento cirúrgico \\
\hline 3. Medidas gerais (instruções ao paciente) \\
\hline 4. Medidas compensatórias para a deglutição (espessantes, posturas e manobras) \\
\hline 5. Reabilitação da deglutição \\
\hline 6. Sonda nasogástrica/nasoenteral \\
\hline 7. Injeção de toxina botulínica no cricofaríngeo \\
8. Dilatação instrumental do cricofaríngeo \\
9. Tratamento endoscópico do divertículo de Zencker \\
10. Miotomia do cricofaríngeo
\end{tabular}

\section{Reabilitação da deglutição}

Envolve exercícios que treinam músculos específicos ou grupamentos musculares e tem sido eficaz em melhorar a deglutição e reduzir as complicações da DOF. Há exercícios para fortalecer a musculatura da língua, a formação e a homogeneização do bolus, a excursão laríngea e a contração da faringe. Um deles é o exercício de Shaker, com flexão da cabeça em posição deitada, que objetiva o fortalecimento dos músculos supra-hióideos, o aumento da abertura do EES e o movimento anterior da laringe, reduzindo os resíduos pós-deglutição e aspiração.

\section{Outras intervenções}

\section{Sonda nasogástrica ou nasoenteral}

É empregada para pacientes com DOF instalada de forma aguda, para aqueles com disfagia total ou para complementar a hidratação ou nutrição. A sonda nasogástrica é recomendada por curto período (máximo dois meses). Algumas das desvantagens são risco aumentado de refluxo gastroesofágico (RGE), lesões nasais e esofágicas.

\section{Gastrostomia endoscópica percutânea (GEP)}

$\mathrm{Na}$ GEP, uma sonda adequada é colocada no estômago através da parede do abdome. É recomendada em pacientes que necessitam de nutrição enteral em longo prazo (mais de 4 a 6 semanas), como nas doenças crônicas ou progressivas. Pacientes geriátricos com DOF pós-AVC e/ou os com demências são as principais indicações para a GEP. Há controvérsias a respeito da prevenção de aspiração com o uso da GEP e sabe-se que a GEP pode causar ou aumentar o risco de RGE com possibilidade de aspiração. Medidas antirreflu- 
xo podem, portanto, ser necessárias. A GEP deve ser revista periodicamente, com troca de sonda, se necessário.

\section{Injeção de toxina botulínica}

A injeção de toxina botulínica (TB) no músculo cricofaríngeo por via endoscópica tem sido registrada em pequenas séries de casos. Um estudo recen$\mathrm{te}^{2}$ empregou a TB (15 a $\left.20 \mathrm{U}\right)$ em 76 pacientes com doenças neurológicas diversas, nos quais havia sido comprovado falha no relaxamento do EES, sendo que bons resultados foram observados em $52 \%$. Houve pneumonia de aspiração em dois pacientes, que haviam sido submetidos a uma segunda injeção e, por esta razão, os autores são cautelosos em recomendar a segunda aplicação.

\section{Dilatações instrumentais do cricofaríngeo}

A técnica consiste no emprego de dilatadores como as bugias de Hurst lubrificadas, com o paciente sentado. As sondas são introduzidas pela boca através da faringe e transição faringoesofágica, onde se observa resistência variável até o esôfago cervical. Mais de $80 \%$ de 103 pacientes tratados em um período de 4 semanas obtiveram resultado satisfatório no alívio da $\mathrm{DOF}^{3}$. Mais recentemente, um pequeno grupo de portadores de distrofia muscular oculofaringea e DOF foi submetido a dilatações com dilatadores de Savary-Gilliard. Todos tiveram boa resposta, com mediana de acompanhamento de 13 anos e média de 7 dilatações por paciente ${ }^{4}$. Não há estudos controlados utilizando dilatações instrumentais.

\section{Tratamento endoscópico}

Empregado no divertículo de Zenker, esta técnica foi introduzida por pesquisadores brasileiros ${ }^{5}$ e consiste em uma miotomia endoscópica realizada de forma ambulatorial ou com internação de curta permanência. Os resultados são muito bons, com 90 a 100\% dos pacientes assintomáticos após o procedimento. A perfuração é a complicação mais temida, podendo ocorrer em até $15 \%$, necessitando diagnóstico precoce para tratamento adequado.

\section{Tratamento cirúrgico}

Pode ser utilizado em situações específicas, como pacientes com evidências de obstrução no nível do EES, realizando-se a miotomia do cricofaríngeo. Preditores de bons resultados seriam uma iniciação da deglutição intacta, preservação de força lingual e faríngea, elevação adequada do hioide e evidência radiológica ou manométrica de aumento da resistência ao fluxo pelo EES. Embora não haja estudos controlados, informações disponíveis sugerem uma taxa de resposta de $60 \%$ com mortalidade de 1 a $2 \%$. A avaliação pré-operatória deve incluir documentação de associação ou não com RGE. 


\section{CONSIDERAÇÕES FINAIS}

A DOF é uma síndrome complexa de abordagem multiprofissional. Seu diagnóstico baseia-se nas manifestações clínicas e na frequente associação com doenças neurológicas. Em virtude da faixa etária acometida e da associação de comorbidades, a investigação apropriada deve ser feita o quanto antes, para que o tratamento correto seja realizado.

\section{REFERÊNCIAS BIBLIOGRÁFICAS}

1. Monteiro L, Lemme E, Abrahão-Junior LJ, et al. Videofluoroscopia da deglutição - experiência do HUCFF-UFRJ. J Bras Gastroenterol. 2011;(supl 1): 9.

2. Zaninotto G, Marchese Ragona R, Briani C, Costantini M, Rizzetto C, Portale G, et al. The role of botulinum toxin injection and upper esophageal sphincter myotomy in treating oropharyngeal dysphagia. J Gastrointest Surg. 2004;8(8):997-1006.

3. Macedo EDF, Carneiro JH, Gomes GF, et al. Dilatation with Hurst bougies for oropharyngeal dysphagia. Dysphagia. 1998;13:131 (abstract).

4. Manjaly JG, Vaughan-Shaw PG, Dale OT, Tyler S, Corlett JC, Frost RA. Cricopharyngeal dilatation for the long-term treatment of dysphagia in oculopharyngeal muscular dystrophy. Dysphagia. 2012;27(2):216-20.

5. Ishioka S, Sakai P, Maluf Filho F, Melo JM. Endoscopic incision of Zenker's diverticula. Endoscopy. 1995;27(6):433-7.

6. Alfonsi E, Retivo DA, Consentino G, De Icco R, Bertino G, Schindler A, et al. Botulinum toxin is effective in the management of neurogenic dysphagia. Clinical-electrophysiological findings and tips on safety in different neurological disorders. Front Pharmacol. 2017;8:80.

7. Baijens LWJ, Clavé P, Cras P, Ekberg O, Forster A, Kolb GF, et al. European Society for swallowing Disorders - European Union Geriatric Medicine Society white paper: oropharyngeal dysphagia as a geriatric syndrome. Clin Interv Aging. 2016;11:1403-28.

8. Cook IJ. Oropharyngeal dysphagia. Gastroenterol Clin N Am. 2009;3:411-31.

9. Costa MMB, Canevaro LV, Azevedo ACP. Análise dosimétrica do método vídeo-fluoroscópico aplicado ao estudo da dinâmica da deglutição. Radiol Bras. 2000;33:353-7.

10. Costa MMB, Monteiro JS. Exame videofluoroscópico das fases oral e faríngea da deglutição. In: Costa M, Castro LP. Tópicos em deglutição e disfagia. Rio de Janeiro: Medsi; 2003. p.273-84.

11. Costa MMB, Moreno MPR. Videomed. Software sem registro de patente desenvolvido pelo Núcleo de Computação Eletrônica da Universidade Federal do Rio de Janeiro. Rio de Janeiro: NCE/UFRJ; 2000.

12. Doria S, Abreu MAB, Buch R, Assumpção R, Nico MAC, Ekcley CA, et al. Estudo comparativo da deglutição com nasofibrolarinngoscopia e videodeglutograma em pacientes com acidente vascular cerebral. Rev Bras Otorrinolaringol. 2003;69(5):636-42.

13. Espetalier F, Fanous A, Aviv J, Bassiouny S, Desuter G, Nerurkar N, et al. International consensus (ICON) on assesssment of oropharyngeal dysphagia. Eur Ann Otorhinolaryngol Head Neck Dis. 2018;135(1S):S17-S21.

14. Kelly JH. Management of upper esophageal sphincter disorders: indications and complications myotomy. Am J Med. 2000;108:43S-46S.

15. Lacau St Guily J, Zhang KX, Périé S, Copin H, Butler-Browne GS, Barbet JP. Improvement of dysphagia following cricopharyngeal myotomy in a group of elderly patients. Ann Otol Rhinol Laryngol. 1995;104(8):603-9.

16. Neltzer N, Kahrilas PJ, Logeman JÁ. Manofluorography in the evaluation of oropharyngeal dysphagia. Dysphagia. 2012;27:151-61. 